\title{
Modelo comunitario de convivencia en educación primaria
}

\section{Community model of coexistence in primary education}

Fecha de recepción: 23/03/2016

Fecha de aceptación: 30/06/2016
Sara Carbonell Sevilla

CEIP Luis Vives de Cullera

Nuria Cantero Rodríguez

Universidad de Jaén

Maria Navarro Sánchez

Universidad de Valencia.

Patricia Melgar Alcantud

Universidad de Girona

\section{resumen/ahstract:}

Las escuelas que son comunidades de aprendizaje trabajan por la superación de la violencia de género siguiendo la línea de socialización preventiva y desarrollando el modelo dialógico de resolución de conflictos. En este artículo, a través de la realización de un estudio de caso en un aula de segundo de primaria, identificamos algunas de las interacciones sociales que están contribuyendo a afrontar situaciones de violencia que ocurren en la escuela, así como transformar las relaciones entre el alumnado. Estas interacciones han sido reflexionadas en el marco de las tertulias literarias dialógicas. Su análisis ha puesto de relieve, como estrategias efectivas para la mejora de la convivencia en las escuelas, romper el silencio frente a la violencia y potenciar la implicación de todo el alumnado en su denuncia y abordaje.

Schools as learning communities overcome gender violence developing the line of preventive socialization and the dialogic model of conflict resolution. In this article, analyzing a case study in a classroom of second grade, we identify some of the social interactions that are contributing to confront situations of violence occurring at school, and transform relations between students. These interactions have been deliberated within the framework of dialogic literary gatherings. The analysis about that has highlighted, as effective strategies to improve coexistence in schools, break the silence against violence and enhance the involvement of all students in their complaint and approach.

\section{palabras clave/keywordls:}

Socialización preventiva, violencia, violencia de género, tertulias literarias dialógicas.

Preventive socialization, violence, gender violence, dialogic literary gatherings.

\section{Modelo comunitario de convivencia en educación primaria}

Lograr el éxito académico de todos los niños y niñas no es una cuestión que deba analizarse únicamente desde las teorías del aprendizaje, si no que resulta clave vincularlo al clima del centro, a la convivencia. Ya que, diferentes investigaciones, han demostrado que un buen clima escolar promueve el éxito y el logro académico (Cornell, Gregory, Huang y Fan, 2013). 
Actualmente, la violencia escolar afecta a un gran número de niños y niñas, y jóvenes. En los Estados Unidos, se estima que aproximadamente el 28\% de los estudiantes de 12 a 18 años sufren algún tipo de intimidación durante el año escolar (Robers, Zhang, Truman y Snyder, 2012). En el estado español, el índice general de maltrato en el tercer ciclo de educación primaria es de del $21 \%$ y disminuye al 14,6\% en la educación secundaria obligatoria, este maltrato responde a tres características: es una acción intencionada, se busca causar daño, miedo, angustia, es una relación asimétrica con desigualdad de poder entre el agresor y la víctima y es recurrente y reiterativa (Arregi y Martínez, 2012).

A pesar de que en los últimos años han surgido un gran número de investigaciones sobre acoso escolar, son pocas las que contemplan la dimensión de género en sus análisis. Esto lleva a que la violencia de género entre los niños y niñas, así como el acoso sexual no sean considerados como tipos específicos de bullying (Tonso, 2002). Este error común obvia que, en muchas ocasiones, las personas que ejercen el bullying y la violencia de género son las mismas. Concretamente aquellas que responden a un modelo de masculinidad tradicional dominante (Flecha, Puigvert y Rios, 2013). Las investigaciones también nos develan la prevalencia respecto a las víctimas. El riesgo de sufrir abusos físicos o sexuales es mayor entre aquellas chicas que han sufrido violencia durante la educación primaria o secundaria (Exner-Cortens, Eckenrode y Rothman, 2013; Smith et al., 2003).

La percepción de la violencia, así como la socialización en la misma, se inician en las primeras edades, toda exposición a la violencia que los niños y niñas experimenten se convierte en parte de la visión del mundo que tienen (Linda, Mayes y Donald, 2003). A su vez, las relaciones afectivo-sexuales que se establecen durante el período adulto están fuertemente condicionadas por el tipo de interacciones que se han establecido durante la niñez y la adolescencia (Aubert, Melgar y Valls, 2011; Melgar y Valls, 2010). Así, desde la primera infancia niñas y niños están adquiriendo aprendizajes que contribuyen a la construcción de sus modelos de atractivo y relaciones (Gómez, 2004).

Por lo tanto, si bien es cierto que la violencia de género como tal suele aparecer en mayor medida en la etapa secundaria, no podemos desvincular acoso escolar y violencia de género. La superación de la violencia de género empieza con el abordaje de sus primeras manifestaciones, esto es del acoso escolar. Por ello, se recomienda que el análisis de la violencia en las escuelas contemple la dimensión de género y más concretamente los procesos de socialización de los niños y niñas (Forbes, Adams-Curtis, y White, 2004; Mahlstedt y Welsh, 2005). En este sentido, la línea de socialización preventiva de la violencia de género (Oliver y Valls, 2004), en la que se enmarca la presente investigación, estudia los procesos de socialización en modelos de atractivo vinculados o no a la violencia, y su repercusión en las relaciones afectivas y en el sufrimiento de violencia de género. Desde esta línea se entiende que la escuela puede contribuir a reproducir un modelo en que la violencia se justifica, quita importancia, o incluso se valora o ensalza a quien la ejerce, o bien ser un agente transformador donde no se tolere ningún tipo de agresión y las personas que ejercen esa violencia sean rechazadas (Valls, Puigvert y Duque, 2008). 


\section{Líneas de trabajo en la prevención de la violencia de género con impacto social}

Numerosos programas se han centrado en contribuir a incrementar el conocimiento básico de la infancia sobre la violencia y reconocer la presencia en su entorno (Meraviglia et al., 2003). Estos programas han demostrado ser de utilidad pero no suficientes para un verdadero cambio de actitud y de clima en las escuelas. Para ir más allá se requiere un ambiente positivo que no tolere ningún comportamiento de acoso, abuso, menosprecio..., y el diseño de una respuesta de las escuelas sistemática frente a estos incidentes (Carney y Merrell, 2001). Estos elementos se destacan en programas como "Green Dot" o "Expect Respect Project".

En este tipo de programas, siguiendo los resultados de investigaciones con gran impacto científico y social (Coker et al., 2011; Flecha, Soler y Sordé, 2015; Oliver, Soler, y Flecha, 2009; Valls, Puigvert, Melgar y Garcia, 2016), el fomento de un clima de no tolerancia hacia la violencia, va acompañado de la intervención a favor de la víctima ante situaciones de violencia que se estén presenciando. Estos programas se proponen avanzar en la superación de la violencia fomentando una actitud proactiva entre los estudiantes, que contribuya a prevenir o frenar las situaciones de violencia que ocurran a su alrededor (McMahon y Banyard, 2012; Padrós, 2014; Shorey et al., 2012).

Así, por ejemplo, el programa Green Dot $^{1}$, con la finalidad de crear espacios libres de violencia, destaca el papel del bystander -testigo-. A ellos y ellas se les insta a abordar activamente las diferentes situaciones problemáticas que ocurran en su centro y saber responder a las mismas. Las decisiones de las y los "bystander" para intervenir dependen de sus actitudes sociales y morales hacia las víctimas, la percepción de situaciones de acoso, y las reacciones emocionales (Thornberg et al., 2012). Para que la intervención de estas personas se dé, en primer lugar, deben identificar esa situación como una situación de violencia. Es decir, interpretarla como un problema. En segundo lugar, ser conscientes de la responsabilidad que tienen en la actuación. Posteriormente, determinar cómo ayudar y, por último, decidir intervenir.

En España, el modelo dialógico de prevención y resolución de conflictos se fundamenta también en las evidencias científicas antes mencionadas, y se enmarca en la línea de socialización preventiva de la violencia de género. Esta actuación parte del diálogo y la participación conjunta de toda la comunidad como herramientas claves para superar las desigualdades (Martin y Tellado, 2012; Oliver, Soler, y Flecha, 2009; Padrós, 2014). Investigaciones sobre la violencia entre iguales han identificado que cuando la participación comunitaria se basa en un enfoque comunicativo, que crea modelos dialógicos, aumenta el éxito en la prevención de la violencia (Martin y Tellado, 2012). En este sentido, el desarrollo del modelo dialógico de prevención y resolución de conflictos implica la elaboración y aplicación de una norma contando con la participación de toda la comunidad. Para ello, se establece un proceso participativo -basado en la ética procedimental-(Padrós, 2014), en el cual se debe transcender el simple registro de votos y dar lugar a la interacción comunicativa (Elster, 1998). Esta interacción comunicativa permite que, a través de los argumentos, se puedan modificar las posturas iniciales de las personas y también aparecer alternativas. Diferentes investigaciones demuestran que aquellas escuelas que trabajan directamente por la preven- 
ción de cualquier tipo de violencia, por ejemplo, a través de la articulación de normas que gestionen el clima del centro, o contando con el apoyo de personas adultas a estudiantes, tienen niveles más bajos de violencia y otras formas de intimidación a estudiantes (Gregory et al., 2010).

En el marco de este modelo, existen también una serie de premisas que impregnan el clima del centro, como son la tolerancia 0 ante cualquier tipo de violencia, o la solidaridad y apoyo a las víctimas. Este último punto, está cobrando especial relevancia en todos aquellos programas que demuestran tener buenos resultados en la superación de la violencia. Estos programas parten de la conceptualización de la violencia en las escuelas como proceso de grupo y, por lo tanto, tal y como ya hemos destacado, se centran en transmitir al alumnado su responsabilidad frente a este tipo de situaciones, promoviendo el apoyo a aquellas personas que son víctimas de la violencia en lugar de reforzar a las personas agresoras (Saarento y Salmivalli, 2015).

Cabe destacar que en Valencia estas evidencias científicas han sido recogidas por la Consellería de Educación, Cultura y Deporte, en la Orden 62/2014 por la que se actualiza la normativa que regula la elaboración de los planes de convivencia en los centros educativos de la Comunidad Valenciana y se establecen los protocolos de actuación e intervención ante supuestos de violencia escolar. Concretamente el artículo 8, entre las medidas de actuación de éxito escolar que se sugieren encontramos el modelo dialógico de prevención de conflictos. Y en el punto "g" destaca Acciones y medidas, generales y específicas, de atención a la diversidad, que generen y fomenten el desarrollo de actitudes inclusivas en el centro y ayuden a crear un clima de respeto entre los miembros de la comunidad educativa, proponiendo actividades dirigidas a educar en la solidaridad y apoyo social a las víctimas, así como tolerancia cero frente a la violencia, desde las edades más tempranas.

\section{Metodología}

La metodología empleada se enmarca dentro de la orientación comunicativa crítica (Gómez, Latorre, Sánchez y Flecha, 2006). Esta orientación parte de que el proceso de investigación implica comprender e interpretar la realidad pero, sobre todo, transformarla mediante la intersubjetividad, reflexión y autoreflexión. Para ello, se promueve durante todo el proceso de investigación el establecimiento de un diálogo igualitario entre personas investigadas e personas investigadoras, intentando con ello que las interpretaciones sean fruto de ese diálogo conjunto, donde las reflexiones son tenidas en cuenta en función de la validez de los argumentos presentados y no de la posición que ocupa quien las formula.

\section{Participantes y procedimiento}

La investigación se ha realizado en un aula de $2^{\circ}$ de primaria de una escuela de infantil y primaria, durante el curso 2014-2015. Se trata de una escuela que trabaja la convivencia tomando como referencia las evidencias científicas expuestas, así como el marco normativo sobre convivencia establecido en Valencia. Dicho centro aplica diferentes actuaciones educativas de éxito (Flecha et al., 2011), entre las que encontramos las tertulias literarias dialógicas (TLD). 
La muestra estuvo compuesta por 20 alumnos y alumnas ( 8 niñas y 12 niños, de 7 y 8 años de edad) y tres adultos (una madre, un padre y la profesora como moderadora) que participaban en las TLD. En el momento de la investigación se encontraban leyendo el libro de la Eneida. Todos ellos y ellas eran conocedores de los principios del aprendizaje dialógico (Flecha, 1997). A su vez, el grupo clase estudiado lleva dos cursos trabajando la Socialización Preventiva de la Violencia de Género, desarrollada por la Community Researchers on Excellence for All (CREA) ${ }^{2}$ (Gómez, 2004; Valls, Puigvert y Duque, 2008), con la finalidad de desarrollar el modelo dialógico de prevención y resolución de conflictos.

Las técnicas de recogida de información, que seguidamente detallaremos, han sido aplicadas en el marco de la actuación de las tertulias literarias dialógicas. Las TLD son una de las actuaciones educativas de éxito analizadas en el proyecto europeo INCLUD-ED (20062011). En ellas se produce una construcción conjunta de conocimiento y significado en base al diálogo con todo el alumnado participante en la tertulia ${ }^{3}$. Se basa en los 7 principios del aprendizaje dialógico (Aubert et al., 2008; Flecha, 1997) y se desarrolla en torno a las mejores creaciones literarias de la humanidad. El funcionamiento de las tertulias consiste en comentar el texto a través de la selección de párrafos por parte de las personas participantes. Para ello es importante respetar el turno de palabra y escuchar todas las aportaciones. Cada una de las personas va exponiendo el párrafo o párrafos que han seleccionado y comentan por qué lo han subrayado, posteriormente se abre un turno de palabra para ir compartiendo ideas, pensamientos y sentimientos sobre la información seleccionada (Pulido y Zepa, 2010; Serrano y Mirceva, 2010). Para la investigación sobre la mejora de la convivencia y superación de la violencia hemos escogido esta actuación porque dicho tema ha estado presente en gran parte de diálogos y reflexiones que el alumnado ha realizado.

La técnica de recogida de información aplicada ha sido la observación comunicativa. Las observaciones comunicativas se han realizado durante 28 sesiones de tertulias literarias -una vez por semana en el área de lengua castellana durante un curso académico-, que nos han permitido presenciar en directo el fenómeno de estudio. En ellas se han combinado los roles de observación y de participación directa -por parte de la maestra en el rol de moderadora e investigadora-.

\section{Resultados}

El diálogo igualitario en las actuaciones referidas a la violencia tiene un papel importante. En el caso de las tertulias literarias dialógicas, el espacio dialógico e igualitario, ofreció un contexto donde el alumnado se sentía seguro y era capaz de ir transformando sus relaciones. No se trataba de recoger las voces de las personas participantes para describir una realidad identificada en el texto, si no de construir una interpretación conjunta y consensuada orientada a la transformación de la propia.

Los resultados que se presentan a continuación se han centrado en el análisis de aquellas actitudes que han logrado frenar agresiones y que, a largo plazo, no hayan vuelto a suceder. Todas ellas son actitudes que los niños y niñas han manifestado en diferentes espacios de la escuela -patio, clase, pasillos...-, pero que han sido reflexionadas en el marco de las TLD. Es decir, que los niños y niñas de 2 o de primaria, en el desarrollo de las TDL, han vinculado

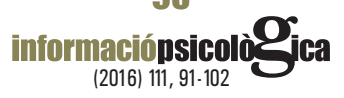


y establecido similitudes entre situaciones de violencia que estaban ocurriendo en el grupo clase y las maneras efectivas de abordarlas, con las reflexiones que les suscitaba el texto.

\section{El buen trato en clase: un problema de todas y todos}

La situación de partida del centro estudiado, respecto a la violencia, se caracterizaba por la existencia de conflictos y el pensamiento generalizado de que éstos no debían abordarse de manera pública. Así por ejemplo, el alumnado, debido a su socialización, asociaba que los conflictos eran algo privado que quedaba entre la profesora, la víctima y el agresor y, por lo tanto, no debían intervenir. -Estaba mal vista la persona que se "metía" en algo que "no era de su incumbencia"-. A su vez, tanto familias como alumnado, verbalizaban que la intervención en situaciones de conflicto conlleva "meterse en problemas".

Investigaciones previas ponen de relieve que frente a las situaciones de violencia se ha establecido una ley del silencio, que tiende a reproducirse debido a que los costes sociales del posicionamiento superan el beneficio de intervenir (Banyard, 2011). Por lo tanto, es común que nadie denuncie este tipo de situaciones, en muchas ocasiones ni siquiera la propia víctima. Invertir esta dinámica requiere que no sea tolerada ninguna situación de violencia, así como no trivializarla. Mostrar una tolerancia cero hacia cualquier tipo de agresión facilita la identificación de las diferentes situaciones de violencia y su denuncia (Valls et al., 2016).

Una de las orientaciones trabajadas ha sido entender las diferentes situaciones de violencia como un problema público. Para ello, en clase, cuando alguien molestaba o agredía a otros -en cualquier espacio- aquellos que querían, iban rechazando públicamente este comportamiento reproduciendo la siguiente frase "no t'ho vaig a permetre" (no te lo voy a permitir) o "no et vaig a permetre que li faces això" (no te lo voy a permitir que le hagas eso). Frases que, poco a poco, el alumnado fue incorporando en sus conversaciones de forma natural.

En el caso del grupo clase estudiado, en el marco de una de las tertulias literarias, la moderadora tuvo que intervenir y parar el debate porque se dio cuenta de que una niña estaba molestando a otra, pero nadie había denunciado la situación. El posicionamiento de la moderadora animó a la niña a que rompiera la ley del silencio y denunciara la situación públicamente, recordándole la importancia de no permitir que nadie la molestara. Esta intervención y reflexión también animó al resto a denunciar una situación que estaba ocurriendo en el comedor escolar, donde la misma alumna molestaba a otros. La víctima se vio reforzada por el grupo y la agresora sintió que todo el grupo rechazaba esa conducta violenta. Algunos niños y niñas reflexionaban la situación y advertían a su compañera del siguiente modo:

Ana: Tú te quedarás sin amigos ¿eh?

María: Tu te quedarás sola como un barco roto.

La implicación del alumnado a lo largo del curso fue aumentando. Conforme fueron sucediéndose las tertulias sus análisis eran más profundos. Pasando de únicamente denunciar situaciones de violencia propias o ajenas, a promover la reflexión conjunta sobre el tipo de amistades que se escogen y qué trato se dan. Estas reflexiones fueron muy clarificadoras para ellos y ellas a la hora de entender todo aquello que no debe permitirse en una relación igualitaria, libre de violencia. A raíz de la lectura de la Eneida continuamente surgían

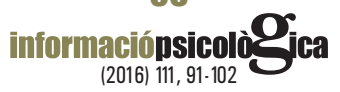


debates relacionados con la violencia, con la guerra, que ofrecían oportunidades para que compartieran aquello que les preocupaba. En una de las conversaciones salió a debate la relación destructiva que tenían dos compañeros de clase, puesto que ellos estaban siempre juntos pero se molestaban o agredían el uno al otro. Gracias a los diálogos de sus compañeros esa relación fue cambiando, convirtiéndose en una relación exenta de violencia. En estas citas se refleja como son capaces de vaciar de atractivo esa relación de violencia y les dicen que no es una relación de amistad:

Antonio: yo no estoy de acuerdo con lo que dice Ernesto porque no puede ser que Álvaro le haga cosas a Marc y después se arrepienta. Se arrepiente y después se lo vuelve a hacer. (...). Si Álvaro te hace una cosa a ti Marc, después tu le haces más cosas a Álvaro, y después tú te quejas Marc, y a ti Álvaro te digo lo mismo.

Laura: es que en el patio siempre estáis juntos a ti siempre te veo con Álvaro, y a ti con Marc. Tenéis que separaros, hasta que Álvaro te trate bien..

Vemos claramente como el grupo se muestra implicado en ayudar a sus compañeros a reflexionar sobre el buen trato y sobre que el tipo de dinámicas que habían establecido no eran saludables.

Tal y como ya hemos mencionado, la propia dinámica de funcionamiento de las TLD pone especial énfasis en el respecto y buen trato. Uno de los principios del aprendizaje dialógico, y que la persona moderadora debe asegurar que se cumple, es el diálogo igualitario. Ello implica que todas las aportaciones sean tenidas en cuenta en función de los argumentos de validez y no en función de la persona que lo diga. En este sentido, se le da mucha importancia a que todos y todas sean escuchados y escuchadas por igual, cosa que no suele ocurrir en las aulas. A no ser que se trabaje de manera específica, es común que se dé mayor importancia a los comentarios de algunas personas, y no tanta a los de otras. En uno de los debates, una niña se dio cuenta que no era escuchado por otro y le dijo públicamente que no le gustaba que no la estuviera escuchando. Fue muy importante que el resto del grupo apoyara a la alumna y se solidarizara con ella en ese momento:

Jorge: no me gusta que trates a Ester mal.

María: yo pienso igual que Jorge, nosotros hemos venido para tratarnos bien, no como se ha comportado Alberto.

Esta conducta fue rechazada por el grupo, que la identificó como un comportamiento no igualitario. Ello contrasta con lo que ocurre si no se trabajan estos temas. Lo que ocurría en este grupo en cursos anteriores era que ese tipo de situaciones y actitudes estaban normalizadas, se les quitaba importancia y, por lo tanto, no se actuaba en contra. Ahora el alumnado es capaz de identificar el mal trato explícito e incluso implícito. En una de las tertulias, se encontraban explicando lo que significa la palabra "enfrentaron", que aparecía en el texto. Sergio intervino y dijo que no lo entendía porque quien hablaba lo hacía en un tono de voz muy bajo, cosa que no era cierta. En otras ocasiones Sergio no había sido sincero, no prestando atención a un compañero y luego diciendo que no lo había podido escuchar . Varias alumnas y alumnos se posicionaron y le dijeron que no les gustaba que no respetara a la persona que hablaba: 
Héctor: no te lo vamos a permitir Sergio.

Eric: no te voy a permitir que le hagas eso a un amigo.

Laura: A ver, no estar atento es una cosa, y decir una mentira es otra. Dices la verdad y ya está. No es tan difícil Sergio.

\section{La fuerza del colectivo}

Sacar a la luz pública situaciones de violencia que ocurren y, especialmente, potenciar el apoyo a las víctimas, permitió que el alumnado diese un segundo paso en sus reflexiones. Éste, fue tomar conciencia del apoyo que supone el colectivo, y las ventajas de abordar las diferentes situaciones con otras personas, en lugar de solos y solas.

Natalia: En esta clase no somos reyes, pero tenemos a todo el resto de la clase para que nos ayude a nosotros en lo que necesitamos.

Esta solidaridad ha promovido el sentimiento de protección. Una protección que proporciona el grupo. Como grupo se sienten a salvo porque siempre van a tener a alguien que les ayude.

Carla: A mí lo que me gusta de este párrafo es que dice reuní a toda mi gente, a mi eso es lo que me gusta. Porque Eneas podía haberse ido solo pero me gusta que pensara en todos y que quisiera irse con su gente, con las personas que él quería. No me gustaría abandonarlas y dejarlas allí, porque juntos pueden conseguir más cosas.

Raúl: Yo pienso como Carla porque a mí me gustaría reunir a toda la gente para salvarlos, no me gustaría coger poquitos.

A su vez, el hecho de sentirse arropadas y arropado por el grupo también les dota de mayor seguridad para afrontar retos y dificultades.

Carlos: Yo me enfrentaría a todo, pero no a los centauros.

Natalia: Yo sola tampoco me enfrentaría, yo sola no. Siempre buscaría amigos y amigas para poder hacer frente a cosas que me pudieran dar miedo.

Dani: cuando estamos juntos...

Inma (mamá): el miedo nos puede paralizar y si estas solo. ¿Qué hacemos? Pero por otra parte cuando hay un grupo de personas entre todos reman para salir adelante (...). Pueden haber dos tipos de miedo. Si estas solo te paraliza, a lo mejor hay personas que sacan fuerzas de ese miedo. Pero hay otras que no pueden, que son más débiles. En cambio, si estamos con más gente pues sacamos fuerzas todos, y aunque uno tenga miedo lo ayudamos. Saca la fuerza y todos vamos juntos.

El hecho de que el alumnado sea consciente de que puede contar con la ayuda, solidaridad e implicación del resto de compañeras y compañeros ha tenido otra consecuencia beneficiosa: dotar de importancia a pedir ayuda. Sabemos que en muchas ocasiones las víctimas de violencia de género viven esta situación en soledad, no explican la situación que están viviendo, guardan silencio. Que el alumnado rompa ese silencio es vital para identificar y destapar todas las situaciones de violencia que ocurren en las escuelas y, ahora mismo, que-

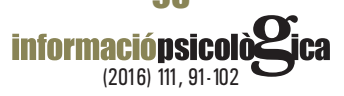


dan invisibilizadas. El siguiente diálogo muestra como el alumnado de este grupo considera muy importante poder pedir ayuda cuando se está en peligro, pero además como valoran positivamente a la persona que se atreve a pedir ayuda:

Alejandro: Si Eneas estuviera en peligro pediría ayuda.

Olga: A mí también me gustaría que me salvaran si estuviera en peligro.

Natalia: Yo también opino como Olga.

Juan: Yo también creo que Eneas es muy valiente por salvar a su padre porque eso es de ser muy valiente y de ser un héroe.

David: Es muy valiente y a mí me gustaría que me salvaran y es muy valiente porque salva a todos, los ayuda.

Silvia: Si tu estuvieras en peligro ¿a quién pedirías ayuda?

Antonio: A mis amigos.

\section{Discusión y conclusiones}

Crear espacios seguros, libres de violencia, es esencial para aumentar el éxito escolar del alumnado. Abordar las primeras manifestaciones de violencia en los centros educativos, constituye el primer paso para la superación de la violencia de género.

La experiencia analizada en este artículo presenta una respuesta eficaz para la mejora de la convivencia en los centros de infantil y primaria, basada en evidencias científicas y que está consiguiendo prevenir la violencia de género desde las primeras edades y contempla el trabajo de casos de violencia de género cuando se den.

La aplicación de la actuación educativa de éxito modelo dialógico de prevención y resolución de conflictos ha supuesto a la escuela estudiada empezar a impregnar el clima escolar $\mathrm{y}$, más concretamente la clase de $2^{\circ}$ de primaria, de una seria de premisas como la tolerancia 0 ante cualquier tipo de agresión o la solidaridad con las víctimas. Los resultados nos muestran una conciencia generalizada de que cualquier situación de violencia no es un problema individual, si no colectivo y, como tal, debe haber una implicación de todos y todas en su denuncia y abordaje.

Para ello, ha sido clave el trabajo en espacios de diálogo transformador, como las tertulias literarias dialógicas, donde a través del diálogo igualitario han ido incorporando la importancia del grupo para prevenir y parar cualquier tipo de agresión.

Con la finalidad de profundizar en el conocimiento sobre las características que están resultando claves para la obtención de sus buenos resultados, sería de interés el desarrollo de futuras investigaciones en otros centros que, siguiendo la línea de socialización preventiva, desarrollen el modelo dialógico de prevención y resolución de conflictos.

El trabajo con niños y niñas desde las primeras edades nos permite soñar con un futuro libre de violencia. Un futuro que, tal y como nos muestra la siguiente cita, también preocupa a las niñas y niños. Los y las del centro estudiado tienen claro que el trabajo que realizan en 
su escuela les está preparando para ese futuro: potenciando la solidaridad y dotándoles de la fuerza de los argumentos.

Sara (reflexionando sobre su futuro paso al instituto): me gustaría en plan... como él es buena persona me hago amigo tuyo, y podemos defendernos mejor los dos juntos, para que no nos hagan daño. Y ayudar a otras personas para que no les hagan daño a ellos. (...). Tenemos que poner todos de nuestra parte. Me gusta cuando dice fuerza, no me gusta si se refiera a fuerza de pegar (...). Nosotros tenemos fuerza, pero no tenemos fuerza de esa de músculos, tenemos la fuerza de la amistad, ¿os acordáis?. Que no tenemos fuerza como para pegar...: «si tú me pegas a mí, yo te pego». Tenemos fuerza como para decirle: «no te vamos a permitir que nos pegues». Nosotros tenemos la fuerza de decir: «no nos gusta que nos pegues ¿cuál es tu argumento?. Y no te lo voy a permitir».

Yo iré a un instituto, que no me peguen, y si me están haciendo daño hablaré con ellos y diré: «¿cuál es tu argumento?» y diré que no se lo voy a permitir

\section{Agradecimientos}

Agradecemos a todas las niñas y niños, familias y profesorado de esta escuela, que ha iniciado el camino hacia una escuela libre de violencia. Con su trabajo puede animar a otras muchas escuelas a transformar sus realidades y hacer posible entornos seguros, y así prevenir la violencia de género.

\section{Referencias}

Arregui, A. y Martínez, P. (2012). El maltrato entre iguales en educación Primaria y ESO. Instituto Vasco de Evaluación e investigación educativa. ISEI-IVEI.

Aubert, A., Flecha, A., García, C., Flecha, R. y Racionero, S. (2008). Aprendizaje dialógico en la Sociedad de la Información. Barcelona: Hipatia Editorial.

Carney, A.G. y Merrell, K.W. (2001). Bullying in Schools: Perspectives on Understanding and Preventing an International Problem. School Psychology International, 22 (3), 364-382.

Coker, A. L., Cook-Craig, P. G., Williams, C. M., Fisher, B. S., Clear, E. R., Garcia, L. S. y Hegge, L. M. (2011). Evaluation of Green Dot: An Active Bystander Intervention to Reduce Sexual Violence on College Campuses. Violence Against Women, 17 (6), 777-796.

Cornell, D., Gregory, A., Huang, F. y Fan, X. (2013). Perceived prevalence of bullying and teasing predicts high school dropout rates. Journal of Educational Psychology, 105, 138-149.

Darley, J.M. y Latené, B. (1968). Bystander Intevention in emergencies. Diffusion of responsability. Journal of Personality and Social Psychology, 4, 377-383.

Elster, J. (1998). Deliberative democracy. Cambridge: Cambridge University Press.

Flecha, A. (2012). Educación y prevención de la violencia de género en menores. Multidisciplinary Journal of Gender Studies, 1 (2), 188211.

Flecha, A., Puigvert, L. y Redondo, G. (2005). Socialización preventiva de la violencia de género. Feminismo, 6, 107-120.

Flecha, R. (1997). Compartiendo palabras. El aprendizaje de las personas adultas a través del diálogo. Madrid, Paidós. 
Duque, E., Flecha, R., García, R., García , P., García, C., Gómez, J., Valls, R. (2011). Actuaciones educativas de éxito en las escuelas europeas. Madrid: Ministerio de Educación.

Exner-Cortens, D., Eckenrode, J. y Rothman, E. (2013). Longitudinal associations between teen dating violence victimization and adverse health outcomes. Pediatrics, 131, 71-78.

Flecha, R., Puigvert, L. y Rios, O. (2013) The New Alternative Masculinities and the Overcoming of Gender Violence. International and Multidisciplinary Journal of Social Sciences 2 (1), 88-113.

Flecha, R., Soler, M. y Sordé, T. (2015). Social impact: Europe must fund social sciences. Nature, 528, 193.

Forbes, G. B., Adam-Curtis, L. E. y White, K. B. (2004). First- and second-generation measures of sexism, rape myths and related beliefs, and hostility toward women. Violence Against Women, 10, 236-261.

Gómez, J. (2004). El amor en la sociedad del riesgo. Barcelona, El Roure.

Gómez, J., Latorre, A., Sánchez, M. y Flecha, R. (2006): Metodología comunicativa crítica.

Barcelona. El Roure.

Gregory, A., Cornell, D., Fan, X., Sheras, P., Shih, T. y Huang, F. (2010). Authoritative school discipline: High school practices associated with lower student bullying and victimization. Journal of Educational Psychology, 102, 483-496.

McMahon, S. y Banyard, V. L. (2012). When can I help? A conceptual framework for the prevention of sexual violence through bystander intervention. Trauma, Violence \& Abuse, 13, 3-14.

Mahlstedt, D. L. y Welsh, L. A. (2005). Perceived causes of physical assault in heterosexual dating relationships. Violence Against Women, 11, 447-472.

Martin, N. y Tellado, I. (2012). Violencia de Género y Resolución Comunitaria de Conflictos en los Centros Educativos. Multidisciplinary Journal of Gender Studies, 1(3), 300-31 9. doi:1 0.4471 /generos. 2012.14

Melgar, P. y Valls, R. (2010). Estar enamorada de la persona que me maltrata: socialización en las relaciones afectivas y sexuales de las mujeres víctimas de violencia de género. Trabajo Social Global, 1 (2), 149-161.

Meraviglia, M.G., Becker, H., Rosenbluth, B., Sanchez, E. y Robertson, T. (2003). The Expect Respect Project - Creating a positive elementary school climate. Journal of Interpersonal Violence, 18 (11), 1347-1360.

Moschella, E., Bennett, S. y Banyard, V. (2016). Beyond the Situational Model: Bystander Action Consequences to Intervening in Situations Involving Sexual Violence. Journal Interpersonal Violence, 1-21.

Linda C., Mayes M.D. y Donald J. (2003). Guía para entender a tu hijo del Centro Yale de estudios infantiles. Un desarrollo sano desde el nacimiento a la adolescencia. Madrid: Alianza editorial.

Oliver, E., Soler, M. y Flecha, R. (2009). Opening schools to all (women): Efforts to overcome gender violence in Spain. British Journal of Sociology of Education, 30, 207-218.

Oliver, E. y Valls, R. (2004): Violencia de género. Investigaciones sobre quiénes, por qué y cómo superarla. Barcelona, El Roure.

Orden 62/2014, de 28 de julio, de la Consellería de Educación, Cultura y Deporte, por la que se actualiza la normativa que regula la elaboración de los planes de convivencia en los centros educativos de la Comunitat Valenciana y se establecen los protocolos de actuación e intervención ante supuestos de violencia escolar. [2014/7282]

Padrós, M. (2014). A Transformative Approach to Prevent Peer Violence in Schools Contributions From Communicative Research Methods. Qualitative Inquiry 20 (7), 916-922.

Pulido, C. y Zepa, B. (2010). La interpretación interactiva de los textos a través de las tertulias literarias dialógicas. Revista Signos, 2, 295-309.

Robers, S., Zhang, J., Truman, J. y Snyder, T.D. (2012). Indicators of School Crime and Safety: 2011. (NCES 2012002/NCJ 236021). National Center for Education Statistics, U.S. Department of Education, and Bureau of Justice Statistics, Office of Justice Programs, U.S. Department of Justice. Washington, DC.

Saarento, S. y Salmivalli, C. (2015). The Role of Clasroom Peer Ecology and Bystanders' Responses in Bullying. Child Development Prespectives, 9, (4), 201-2015. 
Serrano, M. y Mirceva, J. (2010). Dialogic imagination in literacy development. Journal of Psychodidactics 15 (2), 191-205.

Shorey, R. C., Zucosky, H., Brasfield, H., Febres, J., Cornelius, T. L., Sage, C. y Stuart, G. L. (2012). Dating violence prevention programming: Directions for future interventions. Aggression and Violent Behavior, 17, 289-296.

Smith, P. H., White, J. W. y Holland, L. (2003). A longitudinal perspective on dating violence among adolescent and college-age women. American Journal of Public Health, 93, 104-109.

Thornberg, R., Tenenbaum, L., Varjas, K., Meyers, J., Jungert, T. y Vanegas, G. (2012). Bystander Motivation in Bullying Incidents: To Intervene or Not to Intervene?. Western Journal of Emergency Medicine, 13, 3. doi: 10.5811/westjem.2012.3.11792

Tonso, K.L. (2002). Reflecting on Columbine High: Ideologies of Privilege in "Standardized" Schools, Education Studies, 33(4), 389-403.

Valls, R., Puigvert, L. y Duque, E. (2008). Gender Violence amongst teenagers: socialization and prevention. Violence against Women, 14 (7), 759-785.

Valls, R., Puigvert, L., Melgar, P. y Garcia, C. (2016). Breaking the Silence at Spanish Universities: Findings from the First Study of Violence Against Women on Campuses in Spain. Violence Against Women. Recuperado de http://vaw.sagepub.com/reports/most-read

\footnotetext{
https://www.livethegreendot.com/

ii http://crea.ub.edu/index/

iii http://utopiadream.info/ca/actuaciones-de-exito/tertulias-literarias-dialogicas/
} 